\title{
Piezoelectric Polymer Multilayer Coating Method for Vibration Energy Harvester
}

\author{
Nobuaki Kuriyama, ${ }^{1}$ Takashi Nakajima, ${ }^{2,3}$ Ryo Ichige, ${ }^{1}$ and Takaaki Suzuki ${ }^{*}$ \\ ${ }^{1}$ Gunma University, 1-5-1 Tenjin cho, Kiryu, Gunma 376-8515, Japan \\ ${ }^{2}$ Tokyo University of Science, 6-3-1 Niijuku, Katsushika, Tokyo 125-8585, Japan \\ ${ }^{3}$ JST-PRESTO, 4-1-8 Honcho, Kawaguchi, Saitama 332-0012, Japan
}

(Received March 19, 2020; accepted May 7, 2020)

Keywords: piezoelectric, polymer, multilayer coating, $\mathrm{P}(\mathrm{VDF} / \mathrm{TrFE})$, vibration energy harvester, MEMS

In this research, we propose a piezoelectric polymer multilayer coating method by solution casting and spin coating to improve the fabrication yield and piezoelectricity of the vibration energy harvester (VEH). The proposed multilayer coating method improves the smoothness of the surface of the piezoelectric layer and the device fabrication yield. To confirm the validity of the proposed coating method, we fabricated and compared four types of VEHs with different numbers of piezoelectric layers coated on a $\mathrm{Cu}$ /polyimide film. As a result of the evaluation, the piezoelectric characteristics and the fabrication yield of the coated film were found to be improved with increasing number of coating layers. In addition, because of the decrease in the film thickness per coating, the amount of output power generated by the fabricated piezoelectric VEH (PVEH) was increased.

\section{Introduction}

In recent years, energy harvesters have been attracting attention as sensor node power supplies for the Internet of Things (IoT) society. ${ }^{(1)}$ An energy harvester is a power-generation device that captures a small amount of power from the surrounding environment and is used in a self-sustained sensor node in combination with a sensor. The electric power generation methods include that using heat, ${ }^{(2)}$ light, ${ }^{(3)}$ electromagnetism, ${ }^{(4)}$ and vibration. ${ }^{(5,6)}$ In particular, the vibration energy harvester (VEH) is being actively researched because vibration is ubiquitous in the environment and has relatively high energy density. ${ }^{(7)}$ There are several types of VEH, i.e., electrostatic, ${ }^{(8)}$ electromagnetic,${ }^{(9)}$ friction, ${ }^{(10)}$ ionic liquid, ${ }^{(1)}$ and piezoelectric, ${ }^{(12)}$ in the micro-electromechanical systems (MEMS) field. The electrostatic type uses the phenomenon that when a charged object approaches a conductor, a charge of the opposite polarity is generated. The electromagnetic type obtains current from a change in the magnetic flux of a coil caused by a magnet. These types have high flexibility in material selection and device design but require complex microstructures combined with moving and fixed parts. On the other hand, piezoelectric power generation has a simple device structure because electric

*Corresponding author: e-mail: suzuki.taka@gunma-u.ac.jp https://doi.org/10.18494/SAM.2020.2875 
power is generated by strain inside a single material. In addition, the VEH can achieve both device miniaturization and low-frequency vibration response through the use of a highly flexible polymer piezoelectric material. ${ }^{(13)}$ In particular, methods to reduce the overall rigidity of the VEH have been proposed by combining a flexible substrate with an inorganic thin piezoelectric film ${ }^{(14)}$ or by using polymer materials for both the substrate and the piezoelectric element. ${ }^{(15)}$

For a piezoelectric polymer VEH, there are two methods of forming a piezoelectric layer using a commercially available piezoelectric film and a solution casting method. ${ }^{(16-18)}$ In the case of using a film, a device is manufactured by attaching a uniaxially stretched piezoelectric film, which has high piezoelectric performance due to stretching to an elastic layer. ${ }^{(19)}$ However, since a prefabricated film is used, the flexibility of the thickness of the piezoelectric layer is limited in the process. In addition, since the direction of the strain with a high power generation is limited, the characteristics cannot be utilized in a device having a complicated strain-direction distribution. On the other hand, spin coating using a solution in which a piezoelectric material was dissolved with organic solvent in the solution casting method is useful for forming a piezoelectric layer of a device constructed by photolithography. However, the yield of device fabrication is reduced by pinholes that impair insulation and prevent power generation. Furthermore, since the piezoelectricity of the spin-coated layer is inferior to that of the uniaxially stretched film, a sufficient amount of power generation may not be attained.

In this study, we propose a piezoelectric polymer multilayer coating method using solution casting and spin coating to form a piezoelectric polymer VEH. To verify the usefulness of the proposed method, we evaluate the fabrication yield and morphology by atomic force microscopy (AFM) and crystallinity by X-ray diffraction (XRD), and power generation by increasing the number of deposited layers.

\section{Materials and Methods}

\subsection{Fundamental concept of piezoelectric polymer multilayer coating}

The problem with the piezoelectric polymer poly(vinylidene fluoride/trifluoroethylene) $[\mathrm{P}(\mathrm{VDF} / \mathrm{TrFE})]$ formed by the solution casting method is that pinholes are generated in the film. They are caused by particles on the surface of the substrate on which the film is formed and undissolved powder remaining in the solution for dropping, for example. As a result, the top and bottom electrodes for charge acquisition are in contact with each other, and electric power is not generated owing to impaired insulation, as shown in Fig. 1(a). In this study, as shown in Fig. 1(b), to form the piezoelectric layer with insulation, the single coating of film is thinned to reduce the surface roughness, and a multilayer coating is applied to cover and smooth the unevenness. Furthermore, if the thickness of a single layer of $\mathrm{P}(\mathrm{VDF} / \mathrm{TrFE})$ is more than a certain thickness, the molecular chains stand in the direction perpendicular to the substrate, and the piezoelectricity decreases owing to the inability to invert the polarization. ${ }^{(20,21)}$ Therefore, the piezoelectric performance is improved by the crystallinity of each piezoelectric layer upon reducing the thickness of each piezoelectric layer. 


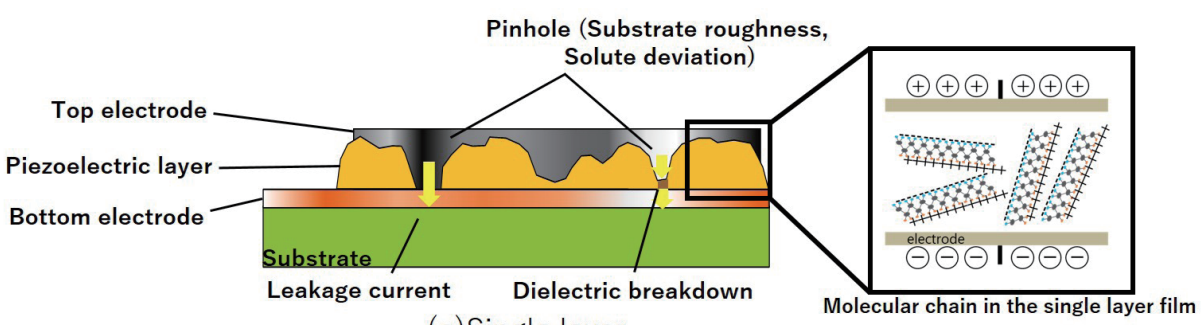

(a) Single layer



Fig. 1. (Color online) Principle of piezoelectric polymer multicoating. (a) Thick single layer coating. (b) Thin multilayer coating.

\subsection{Design and fabrication of piezoelectric VEH (PVEH)}

As shown in Fig. 2, the evaluation device was composed of an elastic layer, a piezoelectric layer, two electrodes, and a proof mass on a typical unimorph cantilever-type ${ }^{(22,23)} \mathrm{PVEH}$. Electrodes for obtaining charge were deposited on the top and bottom of the piezoelectric layer. The device size was set to $10 \times 20 \mathrm{~mm}^{2}$ as the representative dimension similar to that of a button battery. A tungsten proof mass of $0.2 \mathrm{~g}$ was installed on the free end of the cantilever, and the other end was the fixed end attached to the vibration source. When the device is deformed up and down by the acceleration of proof mass and strain is generated in the piezoelectric layer, the spontaneous polarization inside the piezoelectric material changes and charges are generated on the material surface. Since the main purpose of this study was to evaluate the performance of the multilayer-coated piezoelectric film, the device geometry was not optimized to improve the power generation.

As shown in Fig. 3, to simplify the fabrication process of a large number of devices, a $\mathrm{Cu}$ /PI film (Sumitomo Metal Mining Co., Ltd.: S'PERFLEX) serving as an elastic layer with a thickness of $35 \mu \mathrm{m}$ and a bottom electrode with a thickness of $8 \mu \mathrm{m}$ were used for device fabrication. The surface roughness of the $\mathrm{Cu} / \mathrm{PI}$ film is $R q=59 \mathrm{~nm}$, which is sufficiently small compared with the total thickness of the coated piezoelectric layer, so the effect on the surface roughness of the $\mathrm{Cu} / \mathrm{PI}$ film is considered to be small for the piezoelectric layer. The film was cut to a size of $10 \times 30 \mathrm{~mm}^{2}$, and a piezoelectric layer and top electrode were deposited on the $\mathrm{Cu} / \mathrm{PI}$ film to fabricate the device. The $\mathrm{Cu} / \mathrm{PI}$ film was attached and fixed to polydimethylsiloxane (PDMS) (Dow Corning Toray Co., Ltd.: SILPOT 184) formed on a glass substrate (Matsunami Glass Ind., Ltd.: Micro Cover Glass $30 \times 40 \mathrm{~mm}^{2}$ Thickness No. 5). Since the organic material is damaged in the plasma process, ${ }^{(24)}$ the top electrode was coated by vacuum heating vapor deposition (ULVAC KIKO, Inc.: VPC-1100). 


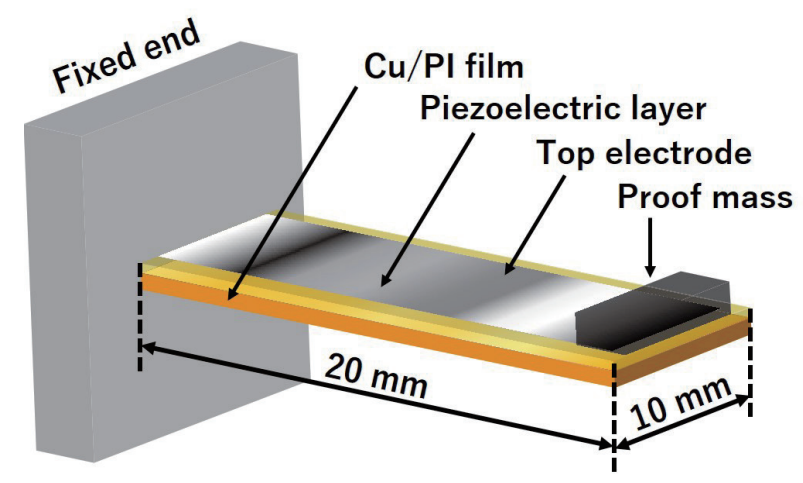

Fig. 2. (Color online) Schematic of fabrication process of solidified ionic liquid. The piezoelectric layer is the flexible organic material $\mathrm{P}(\mathrm{VDF} / \mathrm{TrFE})$ and the elastic layer is made of polyimide.



(1) PDMS spincoating

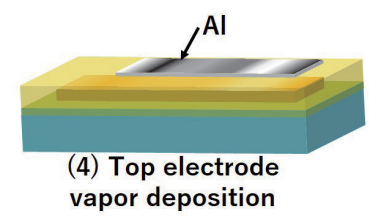

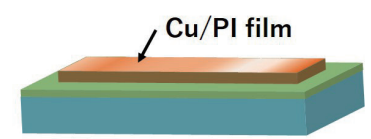

(2) $\mathrm{Cu} / \mathrm{PI}$ film setting



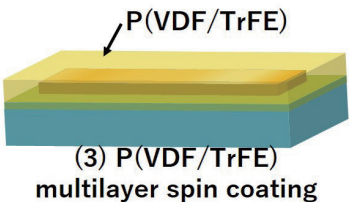



(6) Release and poling

Fig. 3. (Color online) Process flow for PVEH formation. All processes were performed on a single glass substrate.

Table 1 shows the piezoelectric layer coating conditions. The total thickness of the piezoelectric layer was kept constant at $5 \mu \mathrm{m}$ to evaluate the effect of the film thickness per layer and the total number of layers on the piezoelectric performance. The solution used for coating the piezoelectric layer was prepared by dissolving $\mathrm{P}(\mathrm{VDF} / \mathrm{TrFE})$ powder $(75 / 25 \mathrm{~mol} \%)$ (Kureha Corp.: KF Polymer W\#2000) in diethyl carbonate (DEC) (Wako Pure Chemical Corp.: Diethyl Carbonate Wako Special Grade). Before preparing the solution, the powder was dried at $130{ }^{\circ} \mathrm{C}$ for $6 \mathrm{~h}$. The powder and the solvent were mixed in a nitrogen-purged glove box to prevent impurities and moisture. In the multilayer coating method, each layer was baked at $130{ }^{\circ} \mathrm{C}$ for $90 \mathrm{~s}$ for drying and crystallization. After coating the piezoelectric layer and the top electrode, the piezoelectric layer protruding from the $\mathrm{Cu} / \mathrm{PI}$ film was cut off with a cutter and peeled from the glass substrate.

We applied a voltage to the formed piezoelectric layer using a high-voltage DC power supply (Matsusada Precision Inc.: HJPQ-10P3) in polarization processing. The polarization treatment was carried out in vacuum to prevent discharge to the atmosphere and the effect of the external environment. 
Table 1

Deposition conditions of piezoelectric layers.

\begin{tabular}{lrrrr}
\hline Device type & A & B & C & D \\
\hline Piezoelectric layer thickness $(\mu \mathrm{m})$ & 5 & 5 & 5 & 5 \\
Number of layers & 1 & 3 & 5 & 10 \\
Solution concentration $(\mathrm{wt} \%)$ & 20 & 10 & 10 & 5 \\
Rotation speed (rpm) & 7000 & 3000 & 5000 & 1000 \\
\hline
\end{tabular}

\subsection{Experimental setup}

We evaluated the fabrication yield by verifying the insulating properties of the fabricated piezoelectric layer. The percentage of devices completing the polarization process among the devices manufactured under the same conditions is the fabrication yield and was calculated as

$$
\text { Fabrication yield }=\frac{N_{S}}{N_{T}} \text {, }
$$

where $N_{S}$ is the number of successfully fabricated devices, and $N_{T}$ is the total number of fabricated devices. A successfully fabricated device is a device for which polarization has been completed. The completion of polarization was judged from the decrease in the relative dielectric constant after applying the electric field. The alignment of the spontaneous polarization suppresses the polarization fluctuation on applying an $\mathrm{AC}$ electric field, and decreases the dielectric constant. ${ }^{(25)}$ The relative dielectric constant $\varepsilon_{r}$ was calculated using Eq. (2) with the representative dimensions of the device and the capacitance of the piezoelectric layer measured using an LCR meter (NF Corp.: ZM2372).

$$
\varepsilon_{r}=\frac{C d}{\varepsilon_{0} S}
$$

Here, $C$ is the electrostatic capacity of the piezoelectric layer, $d$ is the thickness of the piezoelectric layer, $\varepsilon_{0}$ is the dielectric constant of vacuum, and $\mathrm{S}$ is the area of the top electrode. When the electric field during polarization was $100 \mathrm{MV} / \mathrm{m}$ for $10 \mathrm{~min}$ or more, a piezoelectric performance of $95 \%$ or more was obtained. ${ }^{(26)}$ Devices that became conductive before reaching the target electric field were judged to have failed. The thickness of the piezoelectric layer was measured using a surface roughness measuring device (Tokyo Seimitsu Co., Ltd.: SURFCOM 130A), and the voltage was applied taking into consideration the thickness error of each sample.

To evaluate the film characteristics of the fabricated piezoelectric layer, surface morphology was measured by AFM (Oxford Instruments: MFP-3D) and crystallinity was measured by XRD (Shimadzu Corp.: XRD-6100). The effect of the number of layers on the smoothness of the piezoelectric layer surface was evaluated by AFM. The effects of the film thickness of one layer and the number of film layers on crystallinity were evaluated by XRD. 
The function of the coated piezoelectric layer and the effect of the change in crystallinity on the output power of the device were evaluated by the excitation experiment. For all types of devices used in the test, the total thickness of the coated film was kept constant at $5 \mu \mathrm{m}$ to evaluate the effect of the multilayered piezoelectric layer on power generation with the same strain induced inside the piezoelectric layer. The applied voltage for excitation during the polarization of the device was $60 \mathrm{MV} / \mathrm{m}$. Figure 4 shows the excitation experimental system. The shaker (IMV Corp.: m60/MA1) that applies excitation force to the device, a function generator (NF Corp.: WF1974) that inputs the excitation waveform to the shaker, an acceleration sensor (Micro Stone Corp.: MVP-RF8-HC-2000) for measuring the acceleration on the shaker, an oscilloscope (Teledyne LeCroy: Waverunner 44xi-A) for measuring the output voltage, and a load resistance constitute the experiment system. First, the resonance frequency of the device was specified by gradually changing the excitation frequency of the sine wave input to the shaker. Next, the impedance was matched by changing the external load resistance, and the maximum output power $P_{\max }$ was calculated from the external load resistance and the output voltage as

$$
P_{\max }=\frac{\left(V_{\max }\right)^{2}}{R}
$$

where $V_{\max }$ is the maximum output voltage, and $R$ is the synthetic external load resistance. The internal resistance of the oscilloscope used in the measurement circuit was $1 \mathrm{M} \Omega$. The combined resistance of the connected load resistance and the internal resistance of the oscilloscope was treated as the synthetic external load resistance. ${ }^{(27)}$

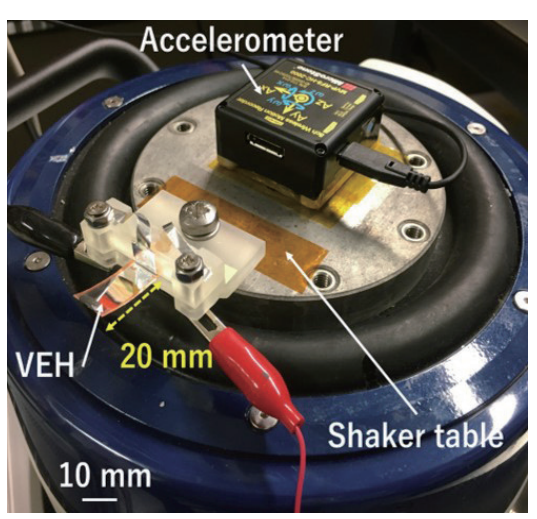

(a)

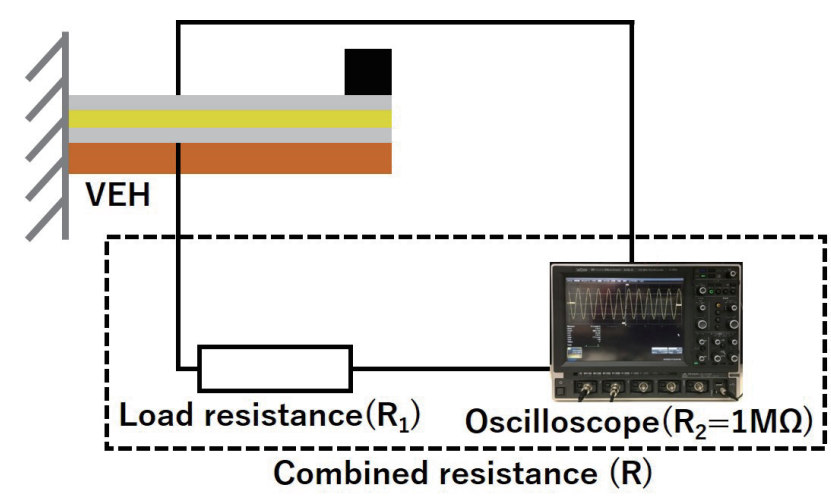

(b)

Fig. 4. (Color online) Excitation experiment system. (a) Photograph of device set up on the shaker. The device electrodes are directly connected to the oscilloscope. (b) Measuring circuit. All elements are connected in series. 


\section{Results and Discussion}

\subsection{Result of fabrication process}

Figure 5 shows the photographs of the fabricated devices. The metallic luster of the surface of the top electrode above the piezoelectric layer improves as the number of deposited layers increases. Since metallic luster reflects the smoothness, the multilayer coating method of the piezoelectric layer reduced the coating nonuniformity, unevenness of solute generated during solvent drying, and roughness due to particles on the substrate. On the surface of the piezoelectric layer observed by AFM, as shown in Fig. 6, the scale of the unevenness of the surface became smaller with increasing number of deposited layers. In addition, Fig. 7 shows the relationship between the root-mean-square (RMS) roughness of the piezoelectric surface obtained from the AFM measurement and the number of deposited layers. Thus, the multilayer coating method is effective for smoothing the surface of the piezoelectric layer. As the number of layers is further increased, a smoother piezoelectric layer is obtained. However, since the number of steps increases with the number of layers in this study, ten coating layers is the most effective from a practical viewpoint.

Figure 8 shows the results measured by XRD. Since the diffraction intensity increased as the number of layers increased, the crystallinity of the film was improved. Although the diffraction intensity per layer decreased as the film became thinner, the total diffraction intensity in the multilayer becomes higher than that of the single layer. In addition, a piezoelectric characteristic of the multilayer was expected owing to the improvement in crystallinity.

Table 2 shows the mean values of the measured electric properties of the piezoelectric layers before and after the polarization treatment. For all device types, the capacitance decreases after polarization treatment, indicating that the relative permittivity decreases from Eq. (2). In addition, the dielectric tangent value is also small, indicating that the loss is low. Thus, the polarization process is complete and the device can be used as a power generation device.

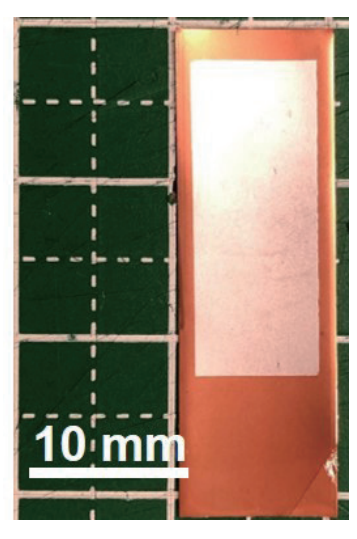

(a)

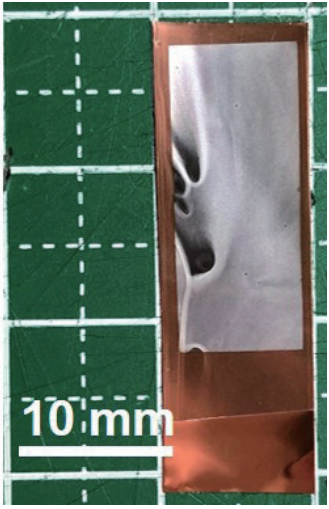

(b)



(c)



(d)

Fig. 5. (Color online) Photographs of the fabricated devices. The piezoelectric layer near the fixed end side was removed to expose the bottom electrode. 




(a)

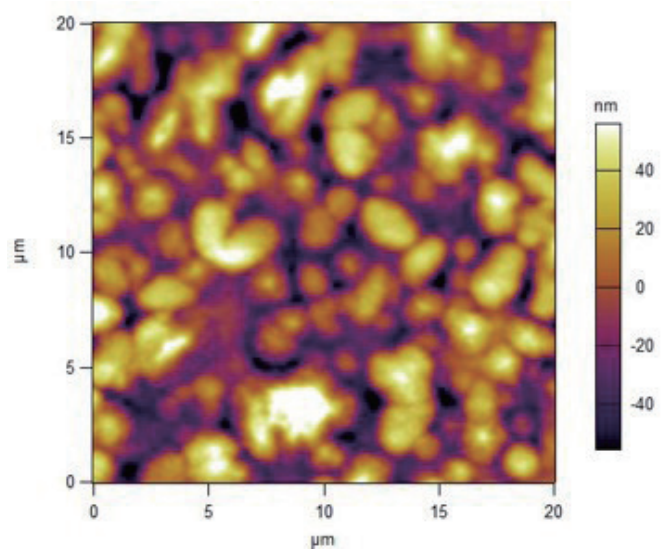

(c)

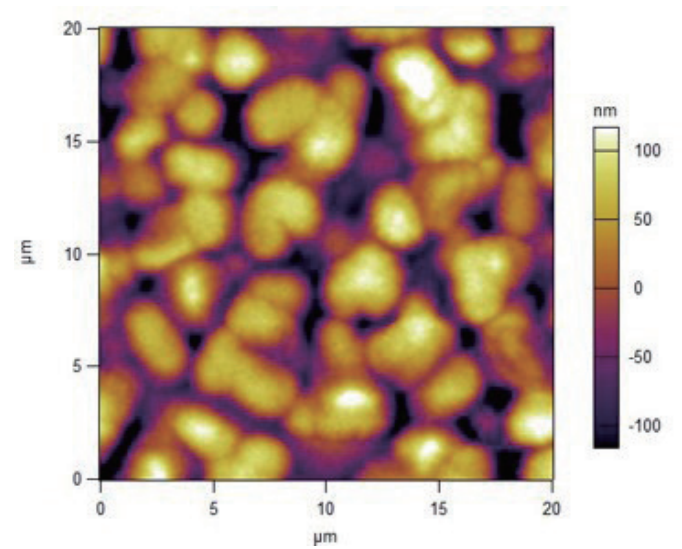

(b)

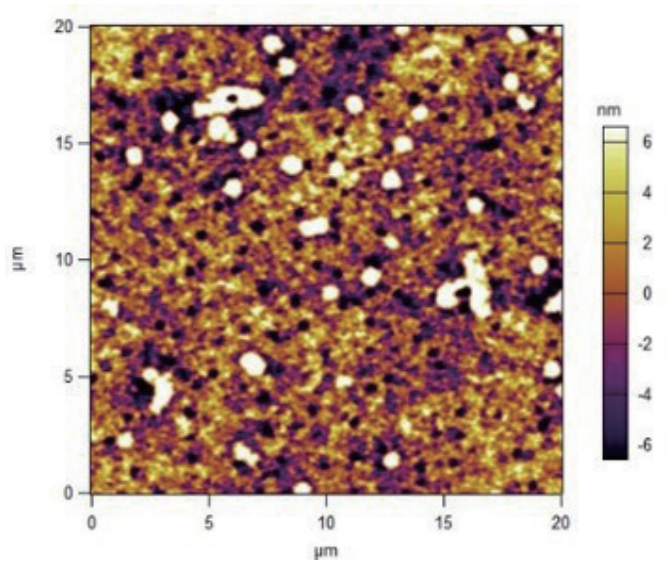

(d)

Fig. 6. (Color online) Results of surface property measurement of the piezoelectric film resurface by AFM. The measurement was performed at a point where no electrode was coated.

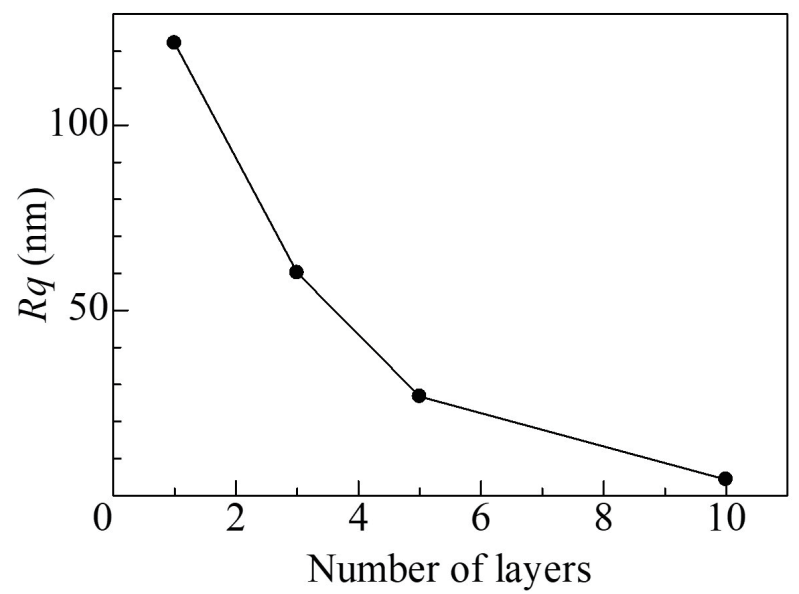

Fig. 7. Relationship between number of layers and RMS of piezoelectric layer surface. The measurement was performed at a point where no electrode was coated. 


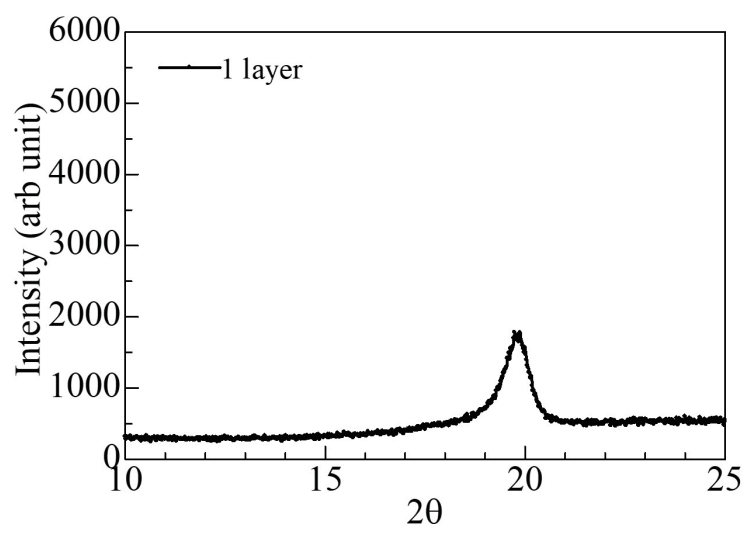

(a)



(c)

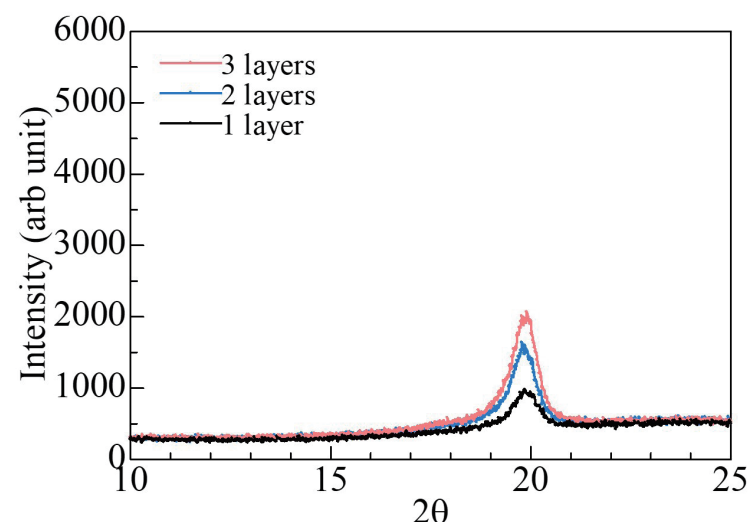

(b)



(d)

Fig. 8. (Color online) Crystallinity of piezoelectric layer measured by XRD. The samples for XRD were directly formed on the glass substrate. The total peak of intensity increases with the number of layers.

Table 2

Changes in electric properties after polarization treatment.

\begin{tabular}{lcccc}
\hline \multirow{2}{*}{ Device type } & \multicolumn{2}{c}{ Before polarization } & \multicolumn{2}{c}{ After polarization } \\
\cline { 2 - 5 } & Capacitance $(\mathrm{nF})$ & Dissipation factor & Capacitance $(\mathrm{nF})$ & Dissipation factor \\
\hline $\mathrm{A}$ & 3.97 & 0.022 & 3.16 & 0.029 \\
$\mathrm{~B}$ & 4.05 & 0.022 & 3.27 & 0.022 \\
$\mathrm{C}$ & 2.90 & 0.019 & 2.18 & 0.021 \\
$\mathrm{D}$ & 3.63 & 0.019 & 2.83 & 0.024 \\
\hline
\end{tabular}

\subsection{Fabrication yield}

Table 3 shows the evaluation results of fabrication yield. The fabrication yield increased by $52.4 \%$ when the number of layers was increased from one to ten layers with a constant total film thickness. As the number of the deposited layers increased, the smoothness of the surface of the piezoelectric layer was improved, unevenness and pinholes of the piezoelectric layer were suppressed, and device failure due to conduction was reduced. In addition, in this evaluation, 
the device fabrication process was simplified by using a $\mathrm{Cu} / \mathrm{PI}$ film precoated with metal. When a metal is coated directly onto a flat substrate, the piezoelectric layer polarization can be expected to be further improved.

\subsection{Power generation}

Figure 9 shows the time history response of the output voltage and the shaker acceleration. Since the output voltage of a device has a waveform synchronized with the input vibration acceleration, the fabricated PVEH responds in accordance with the vibration acceleration.

Figure 10 shows the changes in power generation with the number of layers. The output power from the device increased by $12 \%$ with an increase in the number of film layers, from device A having a single layer to device D having ten layers. Reducing the film thickness per coating layer led to improvements in the piezoelectric property and power generation. The thinning of the piezoelectric layer enabled the polarization reversal of a larger number of molecular chains. The output voltage of the fabricated device was less than 1 V. However, a larger amount of power can be generated by adopting a device shape that makes the distribution of strain uniform or a structure that shows a special deformation behavior, as well as optimizing the thickness of the piezoelectric layer suitable for the strain distribution of each device.

Table 3

Number of layers and device fabrication yield.

\begin{tabular}{lcccc}
\hline Device type & A & B & C & D \\
\hline Fabrication yield (\%) & 33.3 & 60 & 70 & 85.7 \\
Number of samples & 6 & 20 & 10 & 14 \\
\hline
\end{tabular}

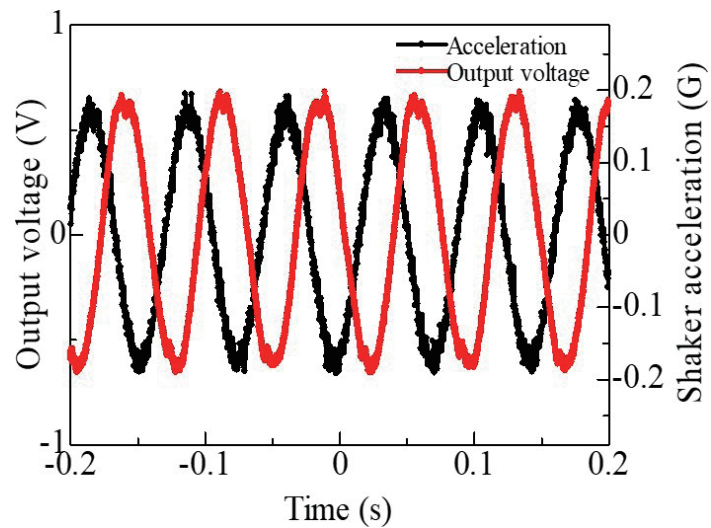

Fig. 9. (Color online) Output voltage of fabricated device D under the following conditions: $0.2 \mathrm{G}$ and $13.8 \mathrm{~Hz}$ frequency.

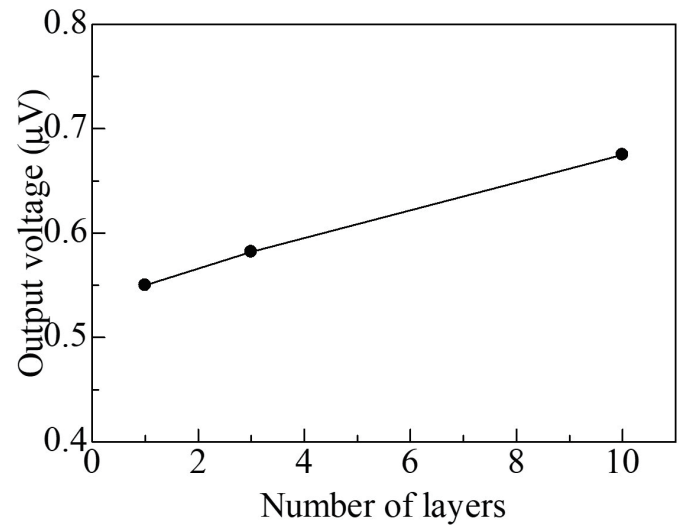

Fig. 10. Power generation plotted against the number of piezoelectric layers in impedance matching at resonance frequency. Proof mass was $0.1 \mathrm{~g}$. Acceleration was $0.2 \mathrm{G}$. 


\section{Conclusions}

We proposed a piezoelectric polymer multilayer coating method using solution casting and spin coating to improve the fabrication yield and piezoelectricity of the piezoelectric layer for VEH. When the piezoelectric layers having a constant total thickness were multilayered, the yield of a 10-layer piezoelectric film was improved by $52 \%$ compared with that of the single-layer piezoelectric film. In addition, the power generation increased by $12 \%$ owing to the decrease in the film thickness per coating layer. By the proposed process, the thickness of the piezoelectric layer is changed in-process, and the manufacturing accuracy of the designed device is improved. The proposed multilayer coating method for the piezoelectric polymer has high compatibility with the MEMS process, and contributes to improving the performance of not only PVEH but also piezoelectric polymer MEMS, such as piezoelectric sensors and actuators.

\section{Acknowledgments}

This research was partially supported by JSPS Science Research Grant JP17H03196, JP20H02095, JST CREST Grant Number JPMJCR19Q2, JST PRESTO Grant Number JPMJPR19R4, Gunma University Regulatory Science Research Grant 2019, and MEXT Nanotechnology Platform Project (The University of Tokyo Microfabrication Platform).

\section{References}

1 S. Priya and D. J. Inman: Energy Harvesting Technologies (Springer, Boston, 2009) 1st ed., p. 3.

2 D. Narducci: J. Phys. Energy 1 (2019) 1. https://doi.org/10.1088/2515-7655/ab0c3a

3 A. Hande, T. Polk, W. Walker, and D. Bhatia: Microprocess. Microsyst. 31 (2007) 420. https://doi.org/10.1016/ j.micpro.2007.02.006

4 K. K. Qureshi, S. Mekid, A. Saleh, and M. S. Sharawi: Sens. Mater. 30 (2018) 1349. https://doi.org/10.18494/ SAM.2018.1830

5 P. D. Mitcheson, G. K. Rao, and T. C. Green: Proc. IEEE 96 (2008) 1457. https://doi.org/10.1109/ jproc.2008.927494

6 H. Toshiyoshi, S. Ju, H. Honma, C. H. Ji, and H. Fujita: Sci. Technol. Adv. Mater. 20 (2019) 124. https://doi.org /10.1080/14686996.2019.1569828

7 S. Roundy, P. K. Wright, and J. Rabaey: Comput. Commun. 26 (2003) 1131. https://doi.org/10.1016/S01403664(02)00248-7

8 P. Basset, D. Galayko, A. M. Paracha. F. Marty, A. Dudka, and T. Bourouina: J. Micromech. Microeng. 19 (2009) 1. https://doi.org/10.1088/0960-1317/19/11/115025

9 Y. N. Chang, H. L. Cheng, S. Y. Chan, and L. H. Huang: Sens. Mater. 30 (2018) 1341. https://doi.org/10.18494/ SAM.2018.1782

10 F. R. Fan, Z. Q. Tian, and Z. L. Wang: Nano Energy 1 (2012) 328. https://doi.org/10.1016/j.nanoen.2012.01.004

11 T. Iida, T. Tsukamoto, K. Miwa, S. Ono, and T. Suzuki: Sens. Mater. 31 (2019) 2527. https://doi.org/10.18494/ SAM.2019.2309

12 G. Li, B. Yang, C. Hou, G. Tang, J. Liu, X. Chen, X. Wang, and C. Yang: Sens. Mater. 29 (2017) 1723. https:// doi.org/10.18494/SAM.2017.1586

13 H. Li, C. Tian, and Z. D. Deng: Appl. Phys. Rev. 1 (2014) 041301-1. https://doi.org/10.1063/1.4900845

14 C. Dagdeviren, B. D. Yang, Y. Su, P. L. Tran, P. Joe, E. Anderson, J. Xia, V. Doraiswamy, B. Dehdasthi, X. Feng, B. Li, R. Poston, Z. Khalpey, R. Ghaffari, Y. Huang, M. J. Slepian, and J. A. Rogers: Proc. Natl. Acad. Sci. 5 (2014) 1927. https://doi.org/10.1073/pnas.1317233111

15 T. Tsukamoto, Y. Umino, S. Shiomi, K. Yamada, and T. Suzuki: Sci. Technol. Adv. Mater. 19 (2018) 660. https://doi.org/10.1080/14686996.2018.1508985 
16 T. Tsukamoto, Y. Umino, K. Hashikura, S. Shiomi, K. Yamada, and T. Suzuki: J. Visualized Exp. 144 (2019) 59067. https://doi.org/10.3791/59067

17 S. Ramasundaram, S. Yoon, K. J. Kim, J. S. Lee, and C. Park: Macromol. Chem. Phys. 210 (2009) 951. https:// doi.org/10.1002/macp. 200800600

18 T. Takiguchi, T. Sasaki, T. Nakajima, S. Yamamura, T. Sekiguchi, and S. Shoji: J. Phys. Conf. Ser. 557 (2014) 1. https://doi.org/10.1088/1742-6596/557/1/012030

19 J. Humphreys, I. M. Ward, J. C. McGrath, and E. L. Nix: Ferroelectrics 67 (1986) 131. https://doi. org/10.1080/00150198608245015

20 D. Guo and N. Setter: Macromolecules 46 (2013) 1883. https://doi.org/10.1021/ma302377q

21 M. A. Barique, M. Sato, and H. Ohigashi: Polym. J. 33 (2001) 69. https://doi.org/10.1295/polymj.33.69

22 G. Tang, B. Bao, Z. Yi, G. Li, B. Yang, and J. Liu: Sens. Mater. 29 (2017) 1733. https://doi.org/10.18494/ SAM.2017.1727

23 S. Saadon and O. Sidek: Energy Convers. Manage. 52 (2011) 500. https://doi.org/10.1016/j.enconman.2010.07.024

24 T. H. Gil, C. May, S. Sholz, S. Franke, M. Toerker, H. Lakner, K. Leo, and S. Keller: Org. Electron. 11 (2010) 322. https://doi.org/10.1016/j.orgel.2009.11.011

25 T. Nakajima, Y. Takahashi, and T. Furukawa: Appl. Phys. A 91 (2008) 33. https://doi.org/10.1007/s00339-0074372-3

26 P. H. Ducrot, I. Dufour, and C. Ayela: Sci. Rep. 6 (2016) 1. https://doi.org/10.1038/srep19426

27 H. C. Song, H. C. Kim, C. Y. Kang, H. J. Kim, S. J. Yoon, and D. Y. Jeong: J. Electroceram. 23 (2009) 301. https://doi.org/10.1007/s10832-008-9439-9

\section{About the Authors}

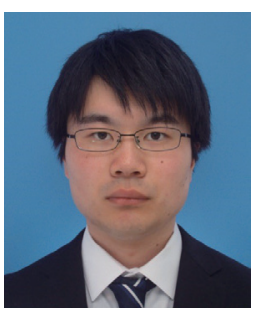

Nobuaki Kuriyama received his B.S. degree from Gunma University, Japan, in 2019. He is now pursuing his M.S degree in the Division of Mechanical Science and Technology at the same university. His research interests are in MEMS and energy harvesting for IoT. (t191b029@gunma-u.ac.jp)

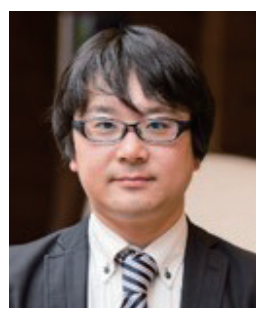

Takashi Nakajima received his B.Sci., M.Sci., and Ph.D. degrees from Tokyo University of Science, Tokyo, Japan, in 2003, 2005, and 2008, respectively. From 2008 to 2012, he worked at Tokyo University of Science as a research associate. From 2012 to 2014, he was at the Institute of Materials Research, Tohoku University, as a research associate. Currently, he is an associate professor in the Department of Applied Physics, Faculty of Science, Tokyo University of Science. He concurrently holds a post of PRESTO researcher of the Japan Science and Technology Agency. His research has been focused on the functional properties of ferroelectric materials and on ferroelectric devices including piezoelectric energy harvesters. (nakajima@rs.tus.ac.jp) 


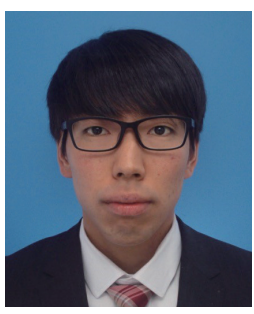

Ryo Ichige received his B.S. degree from Gunma University, Japan, in 2019. $\mathrm{He}$ is now pursuing his M.S. degree in the Division of Mechanical Science and Technology at the same university. His research interests are in MEMS and energy harvesting for IoT. (t191b009@gunma-u.ac.jp)

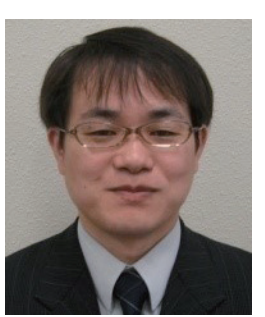

Takaaki Suzuki received his B.E. and M.E. degrees from Gunma University, Japan, in 1998 and 2000, respectively. He received his Ph.D. degree from Kyoto University, Japan, in 2003. From 2004 to 2008, he was an assistant professor at Kyoto University. From 2008 to 2015, he was an associate professor at Kagawa University. From 2015 to 2018, he was an associate professor at Gunma University. Since 2018, he has been a professor at the Division of Mechanical Science and Technology, Gunma University. From 2015 to 2019, he was also a PRESTO researcher at the Japan Science and Technology Agency. His research interests include the development of novel microfabrication technology, microfluidic systems for gene and cell analyses, and energy harvesters for IoT. He is a member of IEEE, the Japan Society of Mechanical Engineers, and the Institute of Electrical Engineers of Japan. (suzuki.taka@gunma-u.ac.jp) 